\title{
A comparison of primary care doctors and dentists in the referral of oral cancer: a systematic literature review
}

Langton S, Cousin GCS, Bankhead CR and Plüddemann A

Mr Steve Langton MSc (Oxon), FDSRCS, FDSRCPS, FRCS*

DPhil student, University of Oxford, UK and Retired Consultant Maxillofacial Surgeon

stephen.langton@kellogg.ox.ac.uk

Mr Gary Cousin MB,ChB(Hons) BDS FDSRCS FDSRCPS FRCS FRCS(OMFS)

Consultant Maxillofacial Surgeon, Royal Blackburn Hospital, Blackburn, UK

gary.cousin@hotmail.co.uk

Professor Clare Bankhead, BSc, MSc, DPhil (Oxon)

Associate Professor, Nuffield Department of Primary Care Health Sciences, University of Oxford, UK clare.bankhead@phc.ox.ac.uk

Dr Annette Plüddemann BSc, MSc, PhD

Nuffield Department of Primary Care Health Sciences, University of Oxford, UK

annette.pluddemann@phc.ox.ac.uk

*Corresponding author

Address for correspondence: Steve Langton 2 Ravensdale Road, Bolton, Lancashire, BL1 5DN stephen.langton@kellogg.ox.ac.uk

Keywords: Oral cancer, cancer referral, comparison of GPs and dentists 


\section{ABSTRACT}

Background: Oral cancer is referred to specialists by both GPs and dentists, with varying proportions reported in different studies. However, some have also noted that dentists more frequently refer oral cancer in the absence of patient-perceived symptoms and may refer oral cancers at an earlier stage. Unfortunately, approximately half the UK adult population do not receive regular dental care.

Objectives: Conduct a systematic review on studies that compare GPs and dentists in the referral of oral cancer focusing on three aspects: (1) proportion of diagnosed oral cancers (2) stage on presentation (3) delay.

Methods: Electronic searches: Medline, Embase, Scopus, Google Scholar, Web of Science, CINAHL. Additional searches: reference lists, authors and conference proceedings.

Results: 22 studies from 10 countries were included, a total of 4953 oral cancers. The percentage of medical referrals ranged from $13 \%$ to $86 \%$, the percentage of dental referrals from $15 \%$ to $80 \%$. Random-effects meta-analysis indicated a combined relative risk of medical/dental referral of 1.36 ( $95 \% \mathrm{Cl} 0.99$ to 1.86). For UK-based studies, the relative risk was also $1.36(95 \% \mathrm{Cl} 1.05$ to 1.76$)$. There was considerable heterogeneity for all studies and for a sub-group of UK studies: $I^{2} 96.4 \%$ ( $95 \% \mathrm{Cl} 95.4$ to 97.1 ) and $81.0 \%$ ( $95 \% \mathrm{Cl} 63.3$ to 90.1 ) respectively. Several studies showed a lower stage for dentally-referred cancers; the combined risk of dentists/GPs referring early (stage 1 and 2) disease was 1.37 (95\% $\mathrm{Cl} 1.17$ to 1.60) and one cause may be the much higher number of cases referred by dentists in the absence of symptoms. No studies demonstrated a statistically significant difference in delay.

Authors' conclusions: Oral cancer is referred by both GPs and dentists, typically about $50 \%$ and $40 \%$ respectively although there is a wide range, probably depending on local circumstances. Both groups require skills in oral examination and lesion recognition and risk factor knowledge.

Effectively, regular dental attenders are a select population gaining a regular screen for oral cancer, yet it is likely that this is not delivered to those with the highest risk. It is suggested that further work is required on accessing high-risk individuals, both for possible screening and preventive interventions. 


\section{INTRODUCTION}

Oral cancer is the sixth most common cancer in the world ${ }^{1}$ and approximately 6500 cases are diagnosed per year in the UK. ${ }^{2}$ The incidence of oral cancer is projected to rise by $33 \%$ in the UK from 2014 to 2035 , with 20 cases per 100,000 people in $2035 .^{3}$

Disease stage at presentation has a major influence on survival ${ }^{4,5}$ and cancers that are larger and have spread to the lymph nodes have a much poorer prognosis. Two-year crude survival for stage 1 (early) disease is approximately $90 \%$ whilst for stage 4 (advanced) disease the rate is less than $50 \%{ }^{6}$ and clearly early diagnosis is very important. Unfortunately, approximately $50 \%$ of oral cancers present at advanced stage 3 or 4 , despite the accessibility of the mouth to relatively easy examination. $^{7}$

Oral cancer is unique amongst malignancies in that it is referred to specialist care by both primary care doctors and dentists, which is unlikely for other types of cancer. ${ }^{8}$ Proportions referred by each group vary across studies. A study of 200 oral cancer patients in the $U^{2}{ }^{2}$ reported that $57 \%$ were referred from their GP, $32 \%$ by their dentist, $2 \%$ via a hospital emergency department and $9 \%$ unknown, whilst in a Canadian series $65 \%$ of oral cancers were referred from a dentist. ${ }^{9}$ In the UK, both medical and dental primary care practitioners are provided with a list of 'guideline' symptoms and signs to assist in the referral of oral cancer. ${ }^{10,11}$ Several authors note the importance of utilising these guidelines in conjunction with thorough history and examination - 'the principles of good medical practice are paramount ${ }^{\prime} .{ }^{10}$ However, one study ${ }^{13}$ found that only $18 \%$ of family physicians carried out an examination of the mouth on half their patients.

Further differences between the referrals from each group of practitioners have been reported. An American study ${ }^{9}$ reported that $47 \%$ of dentist-diagnosed oral cancers were identified during a nonsymptom driven visit whilst all medical referrals were symptom-driven. In the same study, the nonsymptom diagnoses were at a statistically significant lower average clinical and pathological stage (1.7 and 1.6 respectively) than lesions detected during a symptom-directed examination (2.6 and 2.5 respectively). It would seem, therefore, that 'routine examination' of the oral soft tissues at a dental check-up has importance in the early diagnosis of oral cancer and dental practitioners are in a good position to carry out routine soft tissue examination of the oral cavity. ${ }^{14}$ However, English NHS data for 2017-18 indicated that only $50.9 \%$ of adults had visited an NHS dentist in the previous 2 years ${ }^{15}$ and it is probable that those at highest risk of oral cancer are least likely to attend regular dental consultations. ${ }^{16}$ 
The overall aim of this systematic review is to compare primary care doctors and dentists in the referral of oral cancer, focusing on three main objectives: (1) the proportion of diagnosed cancers that were referred by each profession (2) differences in stage on presentation for referrals from each profession (3) differences in delay.

\section{METHODS}

\section{Types of Studies}

Studies that examined the referral of oral cancer and compared GPs and dentists, i.e. the study included data for both professions, were considered for this review. They were required to include one or more of the following outcomes:

(1) Proportion/percentage of diagnosed cancers referred by each profession

(2) Stage on presentation for each profession

(3) Presentation delay in relation to each profession

Studies were required to report on diagnosed oral cancers, rather than suspected cancer referrals. Scoping indicated that most studies were likely to be case series but other designs were acceptable, provided that they reported one or more of the required outcomes. Case reports, economic studies and qualitative studies were excluded. Rare types of intra-oral malignancy (such as lymphoma) were excluded.

\section{Search Methods}

The following electronic resources were searched: MEDLINE, EMBASE, Scopus, Google Scholar, Web of Science, CINHAL to May 2019. Further resources included: browsing the reference lists in initiallyidentified studies, author searching (in relation to initially-identified studies), citation index search, browsing the 'find similar' sections of many electronic databases. In an attempt to identify on-going studies the Research Registry (https://www.researchregistry.com) and database of theses (EThOS Electronic Theses Online Service) were examined. Search terms included: mouth neoplasms, oral cancer and mouth cancer in combination with primary health care, primary care, general practitioner, GP, physicians, dentist, general dental practitioner and GDP. 


\section{Selection of studies}

Following elimination of duplicates, abstracts were assessed to identify papers that included a comparison of primary care doctors and dentists in the referral of oral cancer by a single reviewer (SL). Abstracts indicating that the study did not fulfil the inclusion criteria - for example, qualitative studies or commentaries - were excluded at this stage. For the remaining studies, copies of the full papers were obtained and assessed by two reviewers for inclusion in the systematic review according to the pre-defined criteria.

\section{Data extraction}

Data were extracted by three independent reviewers (SL, CB and GCSC). This was followed by a discussion to cross-check the data and to resolve any discrepancies by jointly re-examining the relevant paper and arriving at an agreement.

Basic extracted data included first author, publication year, study setting, country of origin, study size and study type. Percentages of dental and medical referrals were extracted, together with stage data and data on delay. The authors' main conclusions were noted.

\section{Assessment of study quality}

For the assessment of study quality, the 'MINORS' index ${ }^{17}$ was used, evaluated by a single author. MINORS is a 12-item checklist, 8 items are for non-comparative studies and 4 additional items for comparative studies. Items are 'scored' 2 (reported and adequate), 1 (reported but inadequate) and 0 (not reported). The scale has been used to give an assessment of study quality in a number of systematic reviews to evaluate approximate study quality (0-5 = 'poor', 6-10 = 'fair', 11-16 'good'). ${ }^{18,19}$ However, the use of numerical scales for study quality is often discouraged ${ }^{20}$ and therefore the results are presented as a table to give a general view of quality.

\section{Missing data}

Attempting to obtain missing and/or unclear data, several authors were contacted by email. However, in several instances this was not possible because the required outcome had not been measured or the authors could not respond. Therefore, it was decided to analyse only the available data for each outcome.

\section{Synthesis and analysis of data}

Data synthesis and analysis was carried out with MedCalc@ software version 19.1. Forest plots were generated for relative risk of dental and medical referral. DerSimonian-Laird meta-analysis was 
undertaken, random-effects if $\mathrm{I}^{2}$, was over $60 \%$, fixed effects if less. For stage data, due to the differences in the way results were reported, meta-analysis was carried out for 4 studies, the other results were reported as table and narrative. Delay data were reported as a table and narrative.

\section{Heterogeneity}

There are several potential sources of clinical and methodological heterogeneity in this review including oral cancer rates, access to health professionals and willingness to consult a health professional. Different types of primary to specialist referral systems may be in place and referral patterns may, of course, have changed over the years.

Heterogeneity was estimated by: (1) generation of forest plots and inspection of the $95 \%$ confidence intervals (2) calculation of $\mathrm{I}^{2}$ and associated confidence intervals, estimating the percentage of variation caused by heterogeneity.

\section{Subgroups}

Analysis of subgroups of studies undertaken in the UK was planned for each outcome, if data were available, to identify the effect of studies carried out within the same system of medical care.

\section{RESULTS}

\section{Search outcome}

895 papers were identified by electronic searches, 9 from other sources. Scrutiny of abstracts revealed 32 studies on referral of oral cancer from primary to secondary care. Of these, 22 included data on the referral of oral cancer by primary care doctors and dentists (figure1).

\section{Description of included studies}

22 studies were included in the review ${ }^{9,21-32,33-38,29,2,40}$ (table 1). All 22 studies included data on proportion (percentage) of diagnosed oral cancers that were referred by primary care doctors (possibly referred to as 'general practitioner', 'GP' or 'primary care physician') and dentists. 8 studies compared medical and dental oral cancer referrals in relation to stage on presentation (or an aspect of 'stage' such as primary cancer size). 9 studies included comparative data on delay. 8 studies originated from the UK, 2 from each of USA, Australia and Denmark and 1 from each of Ireland, Canada, Netherlands, Finland, Thailand, Israel, Japan and Argentina. 15 studies were retrospective case series, 1 a prospective case series, 3 were questionnaires, 2 used a combination of case series 
analysis and patients interviews and 1 patient interviews alone. Overall, 4953 oral cancers were included and the number of oral cancers in each study ranged from 20 to 1841

\section{Description of excluded studies}

10 studies were excluded at the 'eligibility' stage ${ }^{41-50}$ (figure 1, supplementary table i). Of these, 6 studies focused on oral cancer knowledge or diagnostic skills of the dental and medical practitioners, 2 studies included suspected oral cancer referrals, rather than diagnosed cancers, in 1 paper the referral sources were unclear and 1 paper reported stage in relation to regularity of dental care, rather than comparing primary care doctors and dentists.

\section{On-going studies}

No on-going studies were identified.

\section{Study quality}

Most studies in this review are retrospective cohort studies, although three studies derived data from questionnaires ${ }^{2,26,30}$ and one from patient interviews ${ }^{35}$. Retrospective cohort studies rely on previously-collected information, in this case from case records and therefore depend on accuracy of collection and recording and therefore are prone to information bias, although this is difficult to quantify in most of the studies in this review. Selection bias may also be a factor - for example in the type of unit collecting the information. One further bias in relation to delay is recall bias; this is particularly the case for patients, for example remembering when symptoms first became evident.

A pragmatic approach was adopted in relation to study quality. Of the included studies, 3 focused specifically on dental/medical referral of oral cancer ${ }^{9,21,32}$. However, most other studies examined oral cancer referral or early diagnosis in relation to a broader range of factors, in which the required outcomes for this review were variables included in the study. Furthermore, studies ranged from 10year population-based studies including almost 2000 patients $^{38}$ to small, audit-type studies ${ }^{39}$. 'MINORS' scores are given in supplementary table ii. Studies scoring over 11 are generally considered to be of 'good' quality.

\section{Effects of the intervention}

\section{Percentages of cases referred by primary care medical and dental practitioners}

There was a wide range of percentages of cases referred by each profession across 22 studies (table 2). Medical referrals ranged from $13 \%{ }^{28}$ to $83 \%^{30}$, dental referrals ranged from $15 \%^{30,32}$ to $80 \%^{28}$. Most oral cancers were referred by primary care doctors and dentists - the mean percentage 
referred by combined 'other' mechanisms (not usually specified but likely to include emergency hospital presentations and referrals from other hospital departments) was $12.2 \%$, range 0 to $31 \%$.

\section{Meta-analysis: relative risk of dental or medical referral}

Meta-analysis was undertaken for the relative risk of medical and dental referral for all studies (figure 2) and UK studies (figure 3). Considerable heterogeneity was evident in all studies $\left(\mathrm{I}^{2}=\right.$ $96.4 \%, 95 \% \mathrm{Cl} 95.4$ to 97.1$)$, slightly less for studies carried out in the UK $\left(\mathrm{I}^{2}=81.0 \%, 95 \% \mathrm{Cl} 63.3\right.$ to 90.1).

The overall pooled (random effects) risk ratio for medical compared to dental referral was 1.36 (95\% $\mathrm{Cl} 0.99$ to 1.86$)$; for UK studies the pooled risk ratio was almost identical, 1.36 (95\% $\mathrm{Cl} 1.05$ to 1.76).

Stage of oral cancer on referral - comparison between referrals from primary care doctors and dentists

8 studies compared oral cancer referrals from primary care doctors and dentists in relation to stage on referral ${ }^{9,21-23,25,2729,35}$ (table 3). Stage data were reported in several different ways including comparing primary tumour $(\mathrm{T})$ stage, specific groupings of $\mathrm{T}$ stage, overall stage and nodal involvement, which precluded formal meta-analysis of all studies. However, 5 studies did show a statistically significant difference in relation to stage on presentation, all demonstrating a greater proportion of late disease being referred by doctors ${ }^{9,21,22,25,27}$. Furthermore, one study ${ }^{9}$ showed an increased proportion of patients with metastatic node disease for cancers referred by primary care doctors. 3 studies showed no statistically significant difference between doctors and dentists in

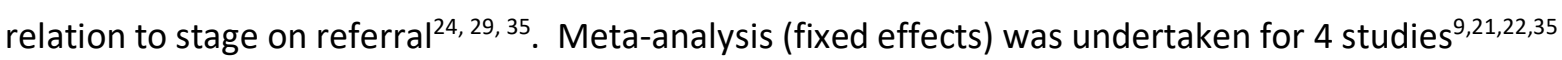
reporting data similarly. The combined risk of dentists/GPs referring early (stage 1 and 2) disease was 1.37 ( $95 \% \mathrm{Cl} 1.17$ to 1.60$), \mathrm{I}^{2} 0.0 \%$ (95\% $\mathrm{Cl} 0.0$ to 78.3 ) - figure 4.

\section{Delay on presentation}

8 papers compared delay for referrals from primary care doctors and dentists $21,22,23,28,29,33,34,37$ (table 4). 'Professional' delay was assessed in 5 studies, 'appraisal period' (patient delay) was measured in 2 studies and the type of delay was not specified in 1 further study. There was no consistency in the method of assessment of delay across studies and formal meta-analysis was not possible. Overall, 2 studies ${ }^{23,37}$ reported no difference in delay whilst 6 reported slightly increased delay for dentists' referrals ${ }^{21,22,29,33,34,37}$ No studies reported a difference of statistical significance. 


\section{Other reported findings}

Several studies reported on the number/proportion of oral cancer cases referred following 'incidental' or 'symptomless' identification at routine examination. In one Dutch study, ${ }^{24} 7$ (14\%) of a series of 50 cancers were diagnosed at routine dental examination. An Australian study of 51 oral cancers $^{25}$ noted that 11 cases (22\%) were identified as 'incidental' findings. A further study ${ }^{9}$ found that of 51 oral cancers, 18 (35\%) of cases were referred on a non-symptomatic basis. Nonsymptomatic cases were associated with a significantly smaller lesion $(p=0.03)$ and cancer stage $(p$ $=0.007)$. A UK study ${ }^{35}$ found that oral cancer identified at 'a routine check-up' was more likely to be at early stage (stage $1 \& 2$ ) than oral cancer referred following symptomatic presentation. Canadian research ${ }^{38}$ found that dentists were more likely to detect asymptomatic cases than primary care doctors (15\% vs $1.4 \%, p<0.0001)$.

\section{DISCUSSION}

\section{GP and dentist referrals}

This review confirms that both primary care doctors and dentists have an important role in the referral of oral cancer to specialist services. Meta-analysis of GP/dentist referral risk ratio indicated that approximately 14 oral cancers are referred from GPs for every 10 from a dentist although there was considerable variation between studies undertaken in different countries - and indeed between studies in the same country. Subgroup analysis of UK studies similarly demonstrated varying proportions of dental and medical referrals, albeit with a smaller range.

The results of these meta-analyses do, of course, need to be interpreted in the light of the considerable heterogeneity that exists between studies undertaken in different countries with varying medical, dental and secondary care systems. The context of the study is also an important consideration. For example, one study in this review ${ }^{9}$, which had a notably high proportion of dentally-referred cancers, included referrals from two dental practitioners with a background of training in maxillofacial surgery. Another study ${ }^{22}$, again reporting a high proportion of dentallyreferred cancers, originated from an academic oral medicine department, which may have a different referral 'profile' to a typical oral and maxillofacial or head and neck unit.

Why might patients present initially to one profession or the other? Studies analysing the presentation of lesions in different parts of the mouth have reported that a dentist was more likely to refer an oral cancer in relation to the areas related to the dentition or dentures $23,24,30$. Alternatively, an Australian study ${ }^{40}$ has suggested that the fact that GP consultations attract a 
financial insurance rebate whilst dental consultations do not was a reason why there had been a shift towards initial oral cancer presentations to GPs. A UK study examining the role of general medical practice in the diagnosis of oral cancer' ${ }^{2}$ suggested that 'evidence is emerging that patients with oral symptoms would rather see their GP than their dentist'. This view was supported in a further study on early oral cancer detection in which over $70 \%$ of respondents considered it likely that they would consult a GP compared to $29 \%$ - $45 \%$ who would consult a dentist with symptoms of potential oral cancer ${ }^{51}$. However, patients attending GPs with 'routine' dental problems is a common occurrence and, furthermore, socio-economically deprived patients, the social group more likely to present with oral cancer, are particularly prone to do this. ${ }^{52}$

Although studies have shown that patients do have variable views on whether they would first attend a primary medical or dental practitioner with a variety of oral-facial problems, ${ }^{53}$ one reason probably influencing the choice of practitioner first seen in relation to oral cancer - and possibly the 'elephant in the room' in relation to this issue - is access to dental services. In the UK, for the 2-year period to March $2018,49.1 \%$ of adults had not seen an NHS dentist. ${ }^{15}$ This phenomenon is by no means restricted to the UK and typically impacts on people who have low income, are unemployed and lacking health insurance, ${ }^{54}$ factors also having established associations with oral cancer. ${ }^{55,56}$

\section{Stage on presentation}

As primary care doctors and dentists refer nearly all oral cancer, the question arises whether referrals from each group have different characteristics that may, potentially, translate into different outcomes.

Overall, studies in this review did not provide data on stage in a consistent format which made full statistical meta-analysis inappropriate. For example, one study grouped $T_{2}$ and $T_{3}$ cancers together as an 'intermediate' group, one study reported average clinical and pathological stage and another the median size of the referred cancer. However, the overall pattern was for either a report of 'no statistical difference' or an earlier stage for dentally-referred cancers.

Several factors may account for the differences in stage on presentation between oral cancers referred by primary care doctors and dentists. One reason may be the proportion of oral cancers identified in the absence of symptoms, as 'incidental' findings. Such diagnoses are made much more frequently by dentists, being very rare in primary care medical practice ${ }^{38}$ and evidence exists that an earlier stage on diagnosis is present with individuals who receive regular dental care ${ }^{41}$. In the studies

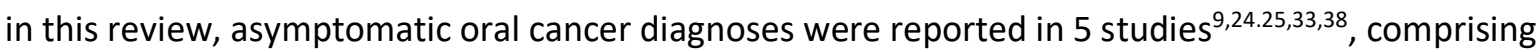
between $14 \%$ and $35 \%$ of cases. In these studies, all except one study reported all asymptomatic 
referrals being from dentists. In one study ${ }^{38} 15 \%$ of dental referrals were asymptomatic compared to $1.4 \%$ of GP referrals.

Studies comparing GPs and dentists in knowledge of oral cancer, pre-malignant lesions, risk factors and oral examination skills and habits indicate dentists are more likely to examine the oral cavity routinely although in one study $94 \%$ of GPs did in response to oral soreness. ${ }^{44,45}$ On the other hand, some argue ${ }^{57}$ that 'the medical practitioner is the most likely resource' for oral cancer screening as doctors are more likely to encounter at-risk patients because of co-existing medical conditions.

This review shows that in a range of different health systems a proportion of oral cancers are, effectively, diagnosed by an 'informal screening procedure' - usually a visit to a dental surgeon - and this, of course links to the rather broader and certainly more complicated question of whether a more systematic, evidence-based approach to screening would be effective. A Cochrane systematic review $^{58}$ provided evidence of reduced mortality, a stage-shift towards earlier diagnosis and reduced costs. However, the reviewers considered that there was insufficient evidence to recommend population-based screening. The Global Oral Cancer Forum ${ }^{59}$ explored the question of screening for oral cancer in considerable detail and reported that accumulated evidence suggested that opportunistic screening of high-risk groups was cost effective but not practical in dental practice as few of the high-risk individuals attended regularly. To date, no screening program for oral cancer has been introduced into the UK. ${ }^{60}$

\section{Differences in delay between medical and dental referrals}

9 studies in this review reported on either 'patient delay' (sometimes referred to as 'appraisal period' delay) or 'professional delay' (practitioner delay). In common with stage of presentation data, there was inconsistency in the method of reporting. For example, studies reported 'mean duration of symptoms', 'mean professional delay', 'median delay' and 'duration of cancer'. Definitions of what period exactly represented delay also varied. For example, one study ${ }^{33}$ defined 'practitioner delay' as being over 6 days delay, whilst other studies ${ }^{34}$ defined 'practitioner delay' as over 2 weeks and this inconsistency made statistical meta-analysis impossible. The overall pattern was one of slightly greater delay for dental referrals, although in no studies was a difference between medical and dental referrals of statistical significance reported. A slightly longer delay in dental referrals has been observed in previous work ${ }^{61}$. Several authors consider one explanation of the difference in delay is that dentists more commonly initiate a 'treatment trial', such as altering dentures prior to referring for secondary care opinion. ${ }^{40,61}$ However, inappropriate initial treatment is not confined to dentists. A study of 779 patients with oral cancer ${ }^{62}$ found $14.8 \%$ had initially 
received inappropriate management, with medical practitioners most often prescribing antibiotics and dentists extracting teeth or adjusting dentures. ${ }^{22}$

Furthermore, the link between diagnostic delay and stage on diagnosis for oral cancer is not clear. It would seem intuitive that delay in diagnosis results in larger cancers and a reduction in survival. However, studies do not point towards a consistent relationship between delay, cancer stage and survival. One study ${ }^{35}$ found no correlation between delay and stage and suggests that 'the error is to assume that symptoms are related to the size a tumour' - in other words, large oral cancers may remain asymptomatic and unnoticed by the patient. However, other work has reported that delayed referral correlated with both more advanced stage at presentation and poorer survival ${ }^{63}$. A metaanalysis found that diagnostic delay was a 'moderate' factor in stage on presentation ${ }^{64}$ although studies on the relationship between delay and stage for oral cancer are not common. ${ }^{65}$

\section{Implications for practice and research}

The findings of our study confirm the important role that both primary care doctors and dentists have in the referral of oral cancer. Guidelines for GPs and dentists in oral cancer diagnosis in the UK are issued by the National Institute of Health and Care Excellence (NICE). The most recent guidelines

${ }^{11}$ advise that lip lumps, erythroplakia or leuko-erythroplakia are referred initially by GPs to a dentist for evaluation. Whilst some work suggests ${ }^{2}$ that increased GP-dentist collaboration is desirable, others consider that such an approach, whilst potentially reducing the secondary care workload, may result in diagnostic delay. This may be worsened by a lack of formal GP-dentist referral systems and insufficient access to dental care for many patients ${ }^{66}$.

The earlier stage of diagnosis by dentists in several studies in this review emphasises the important role of dentists and, potentially, regular dental check-ups in the early diagnosis of oral cancer. However, it is evident that a large section of the population in the UK and elsewhere do not benefit from regular dental care and are often the ones most at risk of oral cancer - the so-called 'inverse screening law'67.

The lack of an oral cancer screening program in the UK continues to be subject of interesting debate. It has been pointed out that the mouth is accessible to simple examination and lesions are relatively easy to detect ${ }^{68}$. Therefore, one might reasonably assume that an oral cancer screening program would be straightforward to put into effect. A systematic review ${ }^{79}$ found 'conventional oral examination' (COE), across 10 studies, gave varying diagnostic accuracy, although sensitivity was at least 0.7 and specificity at least 0.9 in all studies, values of the order delivered by cervical cancer and breast cancer screening programmes. In the only RCT (to date) on oral cancer screening, a large 15 - 
year study carried out in India ${ }^{70}$, a $24 \%$ mortality reduction for high risk individuals was demonstrable. Interestingly, screening was carried out by trained, non-medical, graduates.

It is suggested that further work is required in the identification of high-risk individuals, methods of accessing such people, both for possible screening and preventive interventions, cost-benefit studies and assessment of the use of 'allied healthcare professionals' for oral cancer/pre-cancer screening examinations.

\section{Strengths and weaknesses}

This review provides insight into the referral of oral cancer to specialist care by primary care doctors and dentists, focusing on the proportions referred by each specialty, the differences in stage on presentation and difference in delay, across 22 studies from 12 countries.

However, the included studies did not present data - such as stage - in a consistent format, making formal meta-analysis of some outcomes inappropriate. The long-standing problem of different definitions and methods in studies on early cancer diagnosis research has been addressed by the Aarhus statement ${ }^{\prime 11}$ although this review includes studies undertaken up to 30 years before the Aarhus guidelines were produced. Studies from different countries, and even within the same country, will be influenced by the local systems for referral and organisation of both primary and secondary services. Such differences, together with changes in systems over time, have almost certainly been sources of considerable heterogeneity. The wide range of countries involved does, of course, mean that transferability needs to be interpreted with caution. 
1. Gómez I (2010) Is early diagnosis of oral cancer a feasible objective? Who is to blame for diagnostic delay? Oral Diseases 16(4) 333-342

2. Crossman T et al (2016) Role of general practice in the diagnosis of oral cancer British Journal of Oral and Maxillofacial Surgery 54, pp. 208-212. doi: 10.1016/j.bjoms.2015.11.003

3. Cancer Research UK (2017) Oral cancer incidence statistics available at: ww.cancerresearchuk.org/health-professional/cancer-statistics/statistics-by-cancer-type/oralcancer/incidence

4. Mehanna H, West CML, Nutting C and Paleri V (2010) Head and neck cancer-part 2: treatment and prognostic factors British Medical Journal 341 721-725

5. Mao L, Hong WK and Papadimitrakopoulou VA (2004) Focus on head and neck cancer Cancer Cell 5 311-316

6. South West Cancer Intelligence Unit (2001) Second head and neck audit report (SWAHNII)

7. Stefanuto P, Doucet JC and Robertson C Delays in treatment of oral cancer: a review of the current literature Oral and Maxillofacial Surgery 117 424-429

8. Ford PJ and Farah CS (2013) Early detection and diagnosis of oral cancer: Strategies for improvement Journal of Cancer Policy 1 e2-e7

9. Holmes JD et al (2003) Is detection of oral and oropharyngeal squamous cancer by a dental health care provider associated with a lower stage at diagnosis? Journal of Oral and Maxillofacial Surgery 61(3) 285-91

10. NICE (2004) Improving Outcomes in Head and Neck Cancers - The Manual available at: http://www.nice.org.uk

11. NICE (2015) Suspected cancer: recognition and referral available at: https://www.nice.org.uk/guidance/ng12/chapter/1-recommendations

12. Rubin $\mathrm{G}$ et al (2015) The Lancet Oncology Commission The expanding role of primary care in cancer control Lancet Oncology 16 1231-72

13. Meskin LH (1994) Oral cancer - the forgotten disease Journal of the Amerian Dental Association 125 1042

14. McCann MF, MacPherson LM, Gibson J (2000) The role of the general dental practitioner in prevention and detection of oral cancer; a review of the literature Dental Update 27 404-408

15. NHS Digital (2018) NHS Dental Statistics for England, 2017-18, Second quarterly report available at: https://digital.nhs.uk/data-and-information/publications/statistical/nhs-dental-statistics/nhs-dentalstatistics-for-england-2017-18-second-quarterly-report

16. Donaldson AN et al (2008) The Effects of Social Class and Dental Attendance on Oral Health Journal of Dental Research SAGE 87(1) 60-64

17. Slim KA, Nini E, Forestier D, Kwiatkowski, Panis Y, and Chipponi J (2003) Methodological index for nonrandomized studies (MINORS): development and validation of a new instrument ANZ Journal of Surgery $73712-716$

18. Elkhadem, A., Mickan, S. and Richards, D. (2014) Adverse events of surgical extrusion in treatment for crown-root and cervical root fractures: a systematic review of case series/reports Dental Traumatology 30(1) 1-14

19. Langton S, Siau D and Bankhead C (2016) Two-week rule in head and neck cancer 2000-14: a systematic review British Journal of Oral and Maxillofacial Surgery 54 120-131

20. Higgins JPT, Green S (2011). Cochrane Handbook for Systematic Reviews of Interventions Version 5.1.0 [updated March 2011]. The Cochrane Collaboration. Available from www.handbook.cochrane.org

21. Amsel Z, Strawitz J and Engstrom P (1983) The dentist a source of first episode head and neck cancer patients Journal of the American Dental Association 106 195-197

22. Scully C, Malmos D, Levers BG, Porter SR and Prime SS (1986) Sources and patterns of referrals of oral cancer: role of general practitioners British Medical Journal 293 599-601

23. Schnetler JFC (1992) Oral cancer diagnosis and delays in referral British Journal of Oral and Maxillofacial Surgery 30 210-213

24. Jovanovic A, Kostense PJ, Schulten EAJM, Snow GB, Van Der Waal I (1992) Delay in Diagnosis of Oral Squamous Cell Carcinoma; A Report from The Netherlands European Journal of Cancer. Part B: Oral Oncology 28(1) 37-38

25. Dimitroulis G, Reade P, Wiesenfield D (1992) Referral patterns of patients with oral squamous cell 
carcinoma, Australia European Journal of Cancer Part B: Oral Oncology 28 (1) 23-27

26. Wildt J, Bundgaard T, Bentzen SM (1995) Delay in the diagnosis of oral squamous cell carcinoma Clinical Otolaryngology 20 21-15

27. Gorsky M and Dayan D (1995) Referral delay in diagnosis of oro/oropharyngeal cancer in Israel European Journal of Cancer Part B: Oral Oncology 31(3) 166-168

28. Pinholt EM, Rindum J and Pindborg JJ (1997) Oral cancer: a retrospective study of 100 Danish cases British Journal of Oral and Maxillofacial Surgery 35 77-80

29. Hollows P, McAndrew P and Perini, MG (2000) Delays in the referral and treatment of oral squamous cell carcinoma British Dental Journal 188 262-265

30. Kerdpon D and Sriplung H (2001) Factors related to advanced stage oral squamous cell carcinoma in southern Thailand Oral Oncology 37 216-221

31. Kantola S, Jokinen K, Hyrynkangas K, Mäntyselkä P, and Alho OP (2001) Detection of tongue cancer in primary care British Journal of General Practice 51 106-111

32. O'Sullivan EM Variation in the sources of referral of oral cancer: a population-based study European Journal of Cancer Oral Oncology 37 S49

33. Onizawa K, Nishihara K, Yamagata K, Yusa H, Yanagawa T et al (2003) Factors associated with diagnostic delay of oral squamous cell carcinoma Oral Oncology 39 781-788

34. McLeod NMH, Saeed N and Ali E (2005) Oral cancer: Delays in referral and diagnosis persist British Dental Journal 198 681-684

35. Scott S, Grunfeld E and McGurk M (2005) The idiosyncratic relationship between diagnostic delay and stage of oral squamous cell carcinoma Oral Oncology 41 396-403

36. Morelatto RA, Herrera MC, Fernandez EN et al (2007) Diagnostic delay of oral squamous cell carcinoma in two diagnostic centers in Córdoba Argentina Journal of Oral Pathology and Medicine 36 405-408

37. Rogers S, Pabla R, McSorley A, Lowe D, Brown J et al (2007) An assessment of deprivation as a factor in the delays in presentation, diagnosis and treatment in patients with oral and oropharyngeal squamous cell carcinoma Oral Oncology 43 648-655

38. Groome PA, Rohland SL, Hall SF, Irish J, MacKillop, WJ and O'Sullivan B (2011) A population-based study of factors associated with early versus late stage oral cavity cancer diagnoses Oral Oncology 47 642-647

39. Piggott $L$ (2015) Comparison of the diagnostic yield of medical and dental referrals an oral and maxillofacial 2 weeks rule clinic National Journal of Maxillofacial Surgery 652

40. Kaing L, Manchella S, Love C, Nastri A and Wiesenfeld D (2016) Referral patterns for oral squamous cell carcinoma in Australia: 20 Years progress Australian Dental Journal 61 29-34

41. Elwood JM and Gallagher RP (1985) Factors influencing early diagnosis of cancer of the oral cavity Canadian Medical Association Journal 133 651-656

42. Kowalski L Franco E Torloni H Fava A de Andrade Sobrinho J et al (1994) Lateness of diagnosis of oral and oropharyngeal carcinoma: factors related to the tumour, the patient and health professionals European journal of cancer. Part B, Oral Oncology 30B (3) 167-173

43. Yellowitz J and Goodman $\mathrm{H}$ (1995) Assessing physicians' and dentists' oral cancer knowledge, opinions and practices Journal of the American Dental Association 126 53-60

44. Macpherson L McCann M Gibson J Binnie V and Stephen K (2003) The role of primary healthcare professionals in oral cancer prevention and detection British Dental Journal 195 277-281

45. Carter $L$ and Ogden $G$ (2007) Oral cancer awareness of general medical and general dental practitioners British Dental Journal 203(5) E10

46. Sardella A, Demarosi F, Lodi G, Canegallo L, Rimondi L and Carrassi A (2007) Accuracy of Referrals to a Specialist Oral Medicine Unit by General Medical and Dental Practitioners and the Educational Implications Journal of Dental Education 71 487-491

47. Borhan-Mojabi K Moradi A and Yazdabadi A (2011) Evaluating the degree of knowledge on oral cancer among general practitioners and dentists in Qazvin Journal of Evaluation in Clinical Practice $18498-501$

48. Alami A El Sabbagh R and Hamdan A (2013) Knowledge of oral cancer among recently graduated medical and dental professionals in Amman, Jordan Journal of Dental Education 77 1356-1364

49. Hassona Y Scully C Shahin A Maayta W Sawair F (2016) Factors Influencing Early Detection of Oral Cancer by Primary Health-Care Professionals Journal of Cancer Education 31 285-291

50. Roy S and Anjum K (2018) The two-week wait - a qualitative analysis of suspected head and neck cancer referrals British Dental Journal 225 159-163

51. Eadie D, MacKintosh, MacAskill et al (2009) Development and evaluation of an early detection intervention for mouth cancer using a mass media approach British Journal of Cancer 101 S73-S79 
52. Cope AL, Wood F, Francis NA et al (2015) General practitioners' attitudes towards the management of dental conditions and use of antibiotics in these consultations: a qualitative study BMJ Open 2015 5: e008551. doi:10.1136/ bmjopen-2015-008551

53. Indermun S, Isaacs Q and Mulder R (2017) Participants' preferred choice of practitioner for orofacial symptoms South African Dental Journal 72 315-322

54. Zabos G, Northridge M, Ro M, Trinh C, Vaughan R et al (2002) Lack of Oral Health Care for Adults in Harlem: A Hidden Crisis American Journal of Public Health 92 49-52

55. Greenwood M, Thomson P, Lowry R et al (2003) Oral cancer: Material deprivation, unemployment and risk factor behaviour - An initial study International Journal of Oral Surgery 32 74-77

56. Auluck A, Walker B, Hislop G, Lear S, Schuurman N et al (2016) Socio-economic deprivation: a significant determinant affecting stage of oral cancer diagnosis and survival BMC Cancer 16569

57. Guggenheimer J Weissfeld J and Kroboth F (1993) Who Has the Opportunity to Screen for Oral Cancer? Cancer Causes and Control 4 (1) 63-66

58. Brocklehurst $P$, Kujan O, Glenny A, Oliver R, Sloan P et al (2013) Screening programmes for the early detection and prevention of oral cancer Cochrane Database Syst Rev 2013; CD004150

59. Speight PM, Epstein J, Kujan O, Lingen MW, Nagao T, Ranganathan K et al Screening for oral cancer-a perspective from the Global Oral Cancer Forum Oral Surgery Oral Medicine Oral Pathology Oral Radiology 123 680-687

60. Cancer Research UK (2018) Screening mouth cancer available at: https://www.cancerresearchuk.org/about-cancer/mouth-cancer/getting-diagnosed/screening

61. Grafton-Clarke C, Chen K and Wilcock J (2018) Diagnosis and referral delays in primary care for oral squamous cell cancer: a systematic review British Journal of General Practice DOI: 10.3399/bjgp18X700205

62. Shafer WG (1975) Initial mismanagement and delay in diagnosis of oral cancer Journal of the American Dental Association 90 1262-1264

63. Pitchers M and Martin C (2006) Delay in referral of oropharyngeal squamous cell carcinoma to secondary care correlates with a more advanced stage at presentation, and is associated with poorer survival British Journal of Cancer 94(7) 955-958

64. Seoane J, Takkouche B, Varela-Centelles P, Tomás I and Seoane-Romero J (2012) Impact of delay in diagnosis on survival to head and neck carcinomas: a systematic review with meta-analysis Clinical Otolaryngology 37 99-106

65. Seoane J, Alvarez-Novoa P, Gomez I,Takkouche B, Diz P, Warnakulasiruya S, Seoane-Romero J and Varela-Centelles P (2016) Early oral cancer diagnosis: The Aarhus statement perspective. A systematic review and meta-analysis Head and Neck 38 Suppl 1:E2182-9

66. Grimes D, Patel J and Avery C (2017) New NICE referral guidance for oral cancer; does it risk delay in diagnosis? British Journal of Oral and Maxillofacial Surgery 55 404-406

67. Netuveli G, Sheiham A and Watt RG (2006) Does the 'inverse screening law' apply to oral cancer screening and regular dental check-ups? Journal of Medical Screening 13 47-50

68. Brocklehurst $P$ and Speight $P$ (2018) Screening for mouth cancer: The pros and cons of a national programme British Dental Journal 225 815-819

69. Walsh T, Liu J, Brocklehurst P, Glenny A, Lingen M et al (2013) Clinical assessment to screen for the detection of oral cavity cancer and potentially malignant disorders in apparently healthy adults Cochrane Database of Systematic Reviews 2013 (11) pp: CD010173

70. Sankaranarayanan R, Ramadas K, Thomas G et al. Trivandrum Oral Cancer Screening Study Group (2005) Effect of screening on oral cancer mortality in Kerala, India: a cluster-randomised controlled trial Lancet 365 1927-1933

71. Weller D, Vedsted P, Rubin G et al (2012) The Aarhus statement: improving design and reporting of studies on early cancer diagnosis British Journal of Cancer 106 1262-1267 